\title{
VLA Detections of Stellar Winds Arising from the Galactic Center Arches Cluster
}

\author{
Cornelia C. Lang \\ University of Massachusetts, Amherst, MA 01003, USA \\ W. M. Goss \\ National Radio Astronomy Observatory, Socorro, NM 87801, USA \\ L. F. Rodríguez \\ Instituto de Astronomía, UNAM, Morelia, Michoacán 58089, México
}

\begin{abstract}
VLA detections have been made of 8 sources in the Galactic center Arches cluster. All sources have spectra consistent with those expected for stellar wind emission. Positional correspondence between these sources and near-infrared detections of mass-losing stars confirms that we are detecting stellar wind emission from Arches cluster members.
\end{abstract}

\section{Introduction}

Over the last five years, near-infrared observations have revealed the most dense and massive stellar cluster in the Galaxy (Nagata et al. 1995; Cotera et al. 1996; Serabyn, Shupe \& Figer 1998). The Arches cluster, located $\sim 30$ pc in projection from the Galactic center, and at the center of curvature of a series of filamentary H II regions, is now thought to consist of $>160 \mathrm{O}$-stars within a $<0.5 \mathrm{pc}$ radius (Figer et al. 1999). Initial near-infrared spectroscopy results showed that 12 of the brightest stars are classified as late WN and Of type stars and, based on their emission line widths, are expected to be losing mass at rates of $1-20 \times 10^{-5} \mathrm{M}_{\odot} \mathrm{yr}^{-1}$ (Cotera et al. 1996). The winds arising from such massive stars should be detectable at radio wavelengths, according to the classic theory of Panagia \& Felli (1975) and Wright \& Barlow (1975). In this regime, the emission from ionized, expanding stellar wind envelopes should have a spectral index of $\alpha=+0.6$, where $\mathrm{S}_{\nu} \propto \nu^{\alpha}$.

\section{Observations}

VLA observations of the Arches cluster were made in the A array configuration at $4.9 \mathrm{GHz}$ (4h; July 1999) and $8.3 \mathrm{GHz}$ (4h; July 1999), and in the BnA configuration at $43.3 \mathrm{GHz}$ (3h; October 1999). The phase center of all observations was $\alpha, \delta(2000)=174550.41,-284921.8$, which corresponds to the brightest near-infrared source near the cluster center (Nagata et al. 1995; Cotera et al. 1996). 


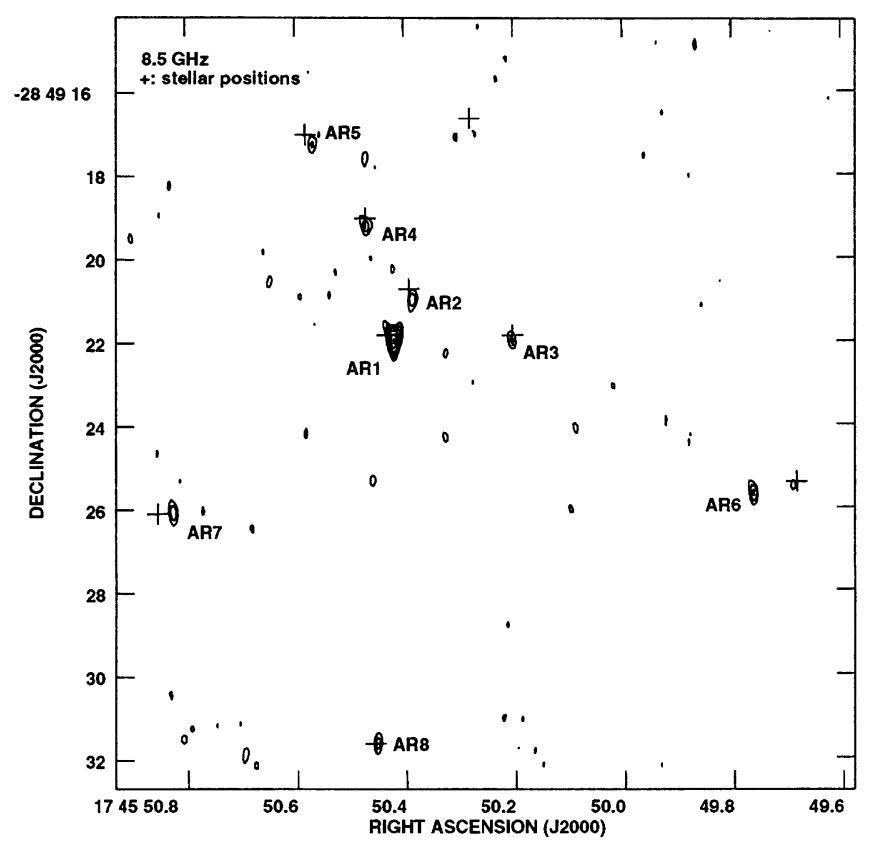

Figure 1. VLA $8.5 \mathrm{GHz}$ continuum image of the inner $16^{\prime \prime}(0.7 \mathrm{pc})$ of the Arches cluster, with a resolution of $0^{\prime \prime} 41 \times 00^{\prime \prime} 16, P A=2{ }^{\circ} 8$. This image has an rms noise of $25 \mu \mathrm{Jy}_{\text {beam }}{ }^{-1}$. Contours correspond to $0.075,0.15,0.25,0.5,1.0,1.5,1.75 \mathrm{mJy} \mathrm{beam}^{-1}$. The crosses in the image show the positions of the near-infrared sources of Nagata et al. (1995) which have been detected in $\operatorname{Br} \gamma$ and $\operatorname{Br} \alpha$ recombination lines.

\section{Results}

At all three frequencies, sources in the Arches cluster were detected. Figure 1 shows the $8.5 \mathrm{GHz}$ image of the radio sources within a $16^{\prime \prime}(0.7 \mathrm{pc})$ region centered on the bright source at the cluster center. The crosses represent the positions of 9 of the 14 stars in Nagata et al. (1995) which exhibit $\operatorname{Br} \gamma$ and $\operatorname{Br} \alpha$ recombination lines and therefore indicate that these stars are losing mass.

The eight sources detected above a $6 \sigma$ level in Figure 1 are referred to as AR1-8. Seven of the sources appear to be associated with the plotted stellar positions. Four of these sources are also detected in the $4.9 \mathrm{GHz}$ observations (AR1, AR4, AR6, and AR7), while AR1 is additionally detected at $43.3 \mathrm{GHz}$. For the sources not detected at $4.9 \mathrm{GHz}$, upper limits for the flux densities at this frequency can be estimated, to produce lower limits for the $4.9 / 8.5 \mathrm{GHz}$ spectral index. The spectral indicies of AR1-8 are listed in Table 1. All but one of the sources show rising spectra in the range of +0.3 to +0.9 , whereas AR6 has a nonthermal spectral index, $\alpha=-0.7$. 
Table 1. Spectral Indicies and Mass-Loss Rates for AR Sources

\begin{tabular}{ccc}
\hline Source & $\alpha(4.9 / 8.3 \mathrm{GHz})$ & $\dot{\mathrm{M}}\left(\mathrm{M}_{\odot} \mathrm{yr}^{-1}\right)$ \\
\hline \hline AR1 & $+0.35 \pm 0.04$ & $1.7 \times 10^{-4}$ \\
AR2 & $>+0.9$ & $3.9 \times 10^{-5}$ \\
AR3 & $>+0.4$ & $3.2 \times 10^{-5}$ \\
AR4 & $+0.65 \pm 0.13$ & $3.9 \times 10^{-5}$ \\
AR5 & $>+0.3$ & $3.0 \times 10^{-5}$ \\
AR6 & $-0.71 \pm 0.08$ & $4.5 \times 10^{-5}$ \\
AR7 & $+0.31 \pm 0.05$ & $4.2 \times 10^{-5}$ \\
AR8 & $>+0.7$ & $3.6 \times 10^{-5}$ \\
\hline
\end{tabular}

\section{Discussion: The Nature of the Arches Radio Sources}

The spectral indicies of the detected sources are consistent with values expected from stellar wind emission. Several of the sources have spectra which are flatter $(\alpha \sim+0.3)$, than the theoretical prediction $(\alpha=+0.6)$, and AR6 has a negative spectral index $(\alpha \sim-0.7)$. However, about $25 \%$ of stellar winds arising from massive stars have been found to have flat or nonthermal spectra in the radio regime (Beiging, Abbott \& Churchwell 1989). The precise mechanism that produces these nonthermal spectra is not established, but internal shocks in the wind of a single star (White 1985) or the presence of interacting winds in close binary systems have been proposed (e.g. Contreras \& Rodríguez 1999). Therefore, the flatter or nonthermal spectra which we observe in some cases are consistent with stellar wind emission from highly evolved supergiants.

Figure 1 also illustrates that seven of the eight radio sources have excellent positional correspondence with the near infrared sources indicated by a cross from Nagata et al. (1995). These stars have all been classified by Cotera et al. (1996) as late-type WN stars, which are expected to be losing mass from their surfaces at high rates. However, the radio source AR6 is displaced by $\Delta \alpha=1^{\prime \prime}$ from the corresponding stellar position of Nagata et al. (1995). There is a $75 \mu \mathrm{Jy} 8.5 \mathrm{GHz}$ source $(3 \sigma)$ closer to the stellar position which may be the correct identification. The stellar source at this position has been classified as a WN8 star by Cotera et al. (1996). AR7 also shows a larger displacement $\left(\Delta \alpha=0^{\prime \prime} 5\right)$ from the corresponding stellar position than the rest of the radio sources. The combination of the radio spectra of these sources and their nearly perfect correspondance with the known mass-losing near-infrared sources overall indicates that for the first we are detecting the stellar wind emission from masslosing stars in the Arches cluster.

We have also determined mass-loss rates (listed in Table 1) from the radio flux densities using the formulation of Panagia \& Felli (1975), assuming a distance to the Galactic center of $8.0 \mathrm{kpc}$ and an electron temperature of $10^{4} \mathrm{~K}$. The mass-loss rates for AR2-8 are in the range 3-4.5 $\times 10^{-5} \mathrm{M}_{\odot} \mathrm{yr}^{-1}$, similar to those rates for other late-type WN systems (Leitherer et al. 1997). However, the mass loss rate of $\mathrm{AR} 1\left(\sim 2 \times 10^{-4} \mathrm{M}_{\odot} \mathrm{yr}^{-1}\right)$ is much larger, and comparable 
only to those observed arising from massive stars in the R136 cluster, which fuels the giant H II region 30 Doradus in the Large Magallenic clouds (de Koter et al. 1997).

The stellar wind detections arising from the Arches cluster are the second stellar wind sources to be discovered in the Radio Arc region. Another of the Galactic center massive clusters, the Quintuplet cluster, is located in the Radio Arc, $\sim 15 \mathrm{pc}$ in projection to the East of the Arches cluster. VLA detections of several stellar wind sources in this cluster were made, and $50 \%$ of them were found to be coincident in position with near-infrared cluster members detected in an HST/NICMOS image (Lang et al. 1999).

This rich population of in massive stars in the Radio Arc suggests that the interstellar environment should be strongly influenced by the energetic processes related to such massive star formation and evolution. Ionization of the Arched Filaments by the Arches cluster and the Sickle H II region by the Quintuplet cluster can be accounted for by the ionizing fluxes of each cluster (Lang et al. 1997; Lang 2000). In addition, interactions between the winds in the densely-packed Arches cluster may give rise to a shock-heated diffuse "cluster wind", which could have a temperature as high as $10^{7} \mathrm{~K}$ (Cantó et al. 2000). This emission is predicted to be detectable in the X-ray regime with the Chandra X-ray Observatory. Studies of the interaction between the massive stars in the Arches and Quintuplet clusters and all components of the surrounding interstellar environoment are currently underway at a number of complimentary wavelengths.

\section{References}

Bieging, J., Abbott, D. \& Churchwell, E. 1989, ApJ, 340, 518

Cantó, J, Raga, A. \& Rodríguez, L.F. 2000, ApJ, 536, 896

Contreras, M. E., Rodriguez, L., \& Gomez, Y. 1996, ApJ, 469, 329

Cotera, A., Erickson, E., Colgan, S., Simpson, J., Allen, D. \& Burton, M. 1996 ApJ, 461, 750

de Koter, A., Heap, S. \& Hubeny, I. 1997, ApJ, 477, 792

Figer, D.F., Kim, S, Morris, M., Serabyn, E., Rich, M., \& McLean, I. 1999, ApJ, 525,750

Lang, C.C., Goss, W.M., \& Wood, D.O.S. 1997, ApJ, 474, 275

Lang, C.C., Figer, D.F., Goss, W.M. \& Morris, M. 1999, AJ, 118, 2327

Lang, C.C., PhD thesis, University of California, Los Angeles

Nagata, T., Woodward, C.E., Shure, M., Kobayashi,N. 1995, AJ, 109, 1676

Panagia, N. \& Felli, M. 1975, A\&A, 39, 1

Serabyn, E., Shupe, D., \& Figer 1998, Nature, 394, 448

White, R.L. 1985, ApJ, 289, 698

Wright, A.E. \& Barlow, M.J. 1975, MNRAS, 170, 41 